\title{
On a modification of the method of Euler polygons for the ordinary differential equation
}

\author{
by A. Pelczar (Kraków)*
}

The subject of the present paper is a problem which has been formulated by Professor T. Ważewski.

§ 1. We shall consider the ordinary differential equation

$$
y^{\prime}=f(x, y)
$$

with the initial condition

$$
y(a)=c .
$$

By $z_{n}(x, \xi), n=1,2, \ldots$, we denote the Euler polygon constructed for the interval $\langle a, \xi\rangle$ and the division $d\left(a_{1}, \ldots, a_{n}\right)$ of this interval by points $a_{j}=a+\frac{j}{n}(\xi-a)(j=0,1, \ldots, n)$. For $n=0$ we define $z_{0}(x, \xi)=c$. Now we put

$$
\varphi_{n}(\xi) \stackrel{\text { df }}{=} z_{n}(\xi, \xi) \text {. }
$$

For example, for the equation

$$
y^{\prime}=y
$$

with the initial condition

$$
y(0)=1
$$

we have $a=0$ and

$$
\begin{array}{ll}
z_{0}(x, \xi)=1, & x \in\langle 0, \xi\rangle, \\
z_{1}(x, \xi)=1+x, & x \in\langle 0, \xi\rangle, \\
z_{2}(x, \xi)= \begin{cases}1+x, & x \in\left\langle 0, \frac{\xi}{2}\right\rangle, \\
\left(1+\frac{\xi}{2}\right)\left(1+x-\frac{\xi}{2}\right), & x \in\left(\frac{\xi}{2}, \xi\right\rangle,\end{cases}
\end{array}
$$

* The author would like to express his sincere thanks to Professor T. Ważewski for his valuable advice. 


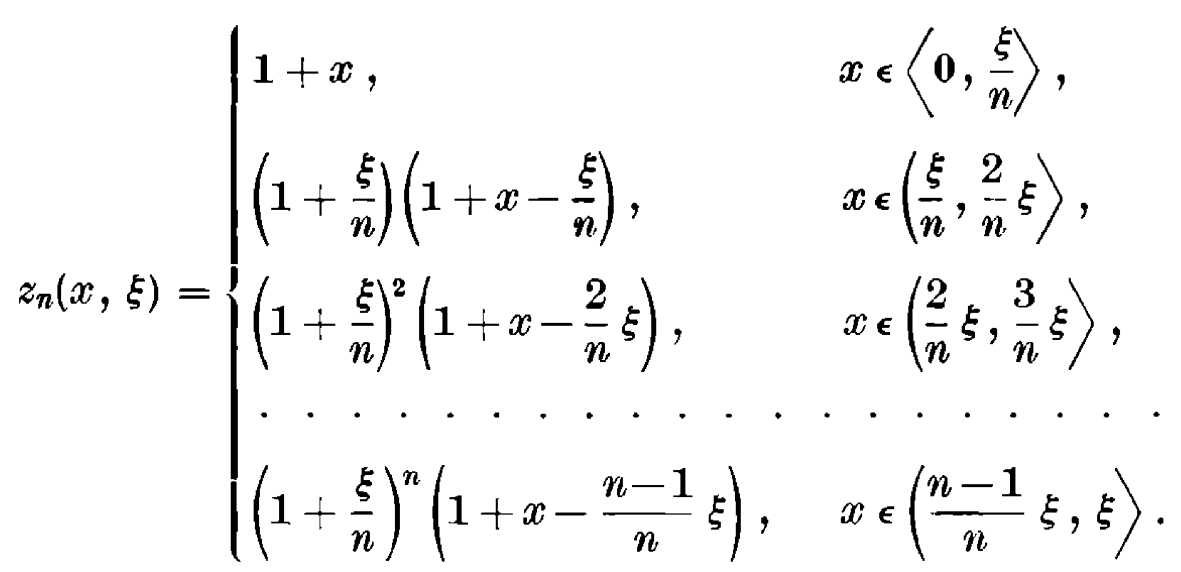

In this case

$$
\varphi_{n}(\xi)=\left(1+\frac{\xi}{n}\right)^{n}
$$

and the sequence $\left\{\varphi_{n}(\xi)\right\}$ is uniformly convergent to $e^{\xi}$ in each interval $\langle 0, b\rangle(b<+\infty)$. Hence for the equation $\left(1.1^{\prime}\right)$ with the initial condition $\left(1.2^{\prime}\right)$, the sequence $\left\{z_{n}(\xi, \xi)\right\}$ is uniformly convergent to the solution of the problem $\left(1.1^{\prime}\right),\left(1.2^{\prime}\right)$. Moreover, in this case, the sequence of the derivatives $\left\{\varphi_{n}^{\prime}(\xi)\right\}$ is uniformly convergent to the derivative of the solution of this problem.

Now the following problem arises: Is the sequence $\left\{\varphi_{n}(x)\right\}$, defined by (1.3), uniformly convergent to the solution of the equation (1.1) with the initial condition (1.2) in the general case?

Remark 1. From the definition of the sequence $\left\{\varphi_{n}(x)\right\}$ it follows directly that we can write it in the following form:

$$
\begin{aligned}
\varphi_{0}(x)=c, \\
\varphi_{n}(x)=\varphi_{n-1}\left(a+\frac{n-1}{n}(x-a)\right)+ \\
+\frac{x-a}{n} f\left[a+\frac{n-1}{n}(x-a), \varphi_{n-1}\left(a+\frac{n-1}{n}(x-a)\right)\right]
\end{aligned}
$$

or equivalently

$$
\begin{aligned}
& \varphi_{0}(x)=c \\
& \varphi_{n}(x)=c+\frac{x-a}{n} \sum_{i=0}^{n-1} f\left[a+\frac{i}{n}(x-a), \varphi_{i}\left(a+\frac{i}{n}(x-a)\right)\right] .
\end{aligned}
$$

The answer to the above problem is given by the following

THEOREM 1. Let us assume that

1. $f(x, y)$ is defined and continuous in the set

$$
\boldsymbol{T}=\{x, y: a \leqslant x \leqslant b,|y-c| \leqslant M\},
$$


2. $|f(x, y)| \leqslant M, b-a<1$,

3. in the interval $\langle a, b\rangle$ there exists exactly one solution $y(x)$ of the problem (1.1), (1.2).

Then the sequence $\left\{\varphi_{n}(x)\right\}$ defined by $(1.3)$ is uniformly convergent to $y(x)$.

Proof. Let us perform the division $d=d\left(\beta_{0}, \beta_{1}, \ldots, \beta_{m}\right)$ of the interval $\langle a, b\rangle$ by the points $\beta_{j}\left(\beta_{j}<\beta_{j+1}\right)$. We put

$$
\delta(d) \stackrel{\text { df }}{=} \max _{j, k=0, \ldots, m}\left(\left|\beta_{j}-\beta_{k}\right|\right) .
$$

By $E(s)$ we denote the set of all Euler's polygons $\psi$ constructed for the interval $\langle a, b\rangle$ and a division $d$ of this interval such that $\delta(d)<s$. We have (cf. [1], III, $\S 8$ )

$$
\mathrm{V}_{\varepsilon>0} \mathbf{H}_{\eta} \nabla_{\psi \in E(s)} \nabla_{x \in\langle a, b\rangle}[s \leqslant \eta \Rightarrow|\psi(x)-y(x)| \leqslant \varepsilon]
$$

where $y(x)$ is the unique solution of the problem (1.1), (1.2). Let $\xi \epsilon\langle a, b\rangle$. By $\hat{z}_{n}(x, \xi)$ we denote the Euler polygon for the division $\hat{d}_{n, \xi}$, which is given by the points

$$
\begin{array}{r}
a, a+\frac{1}{n}(\xi-a), \ldots, a+\frac{n-1}{n}-(\xi-a), \xi, \\
\quad a+\frac{n+1}{n}(\xi-a), \ldots, a+\frac{k}{n}(\xi-a), b
\end{array}
$$

where $k$ is such an integer that

$$
a+\frac{k}{n}(\xi-a) \leqslant b<a+\frac{k+1}{n}(\xi-a) .
$$

Of course, the definition of the sequence $\left\{\hat{z}_{n}(x, \xi)\right\}$ implies directly that

$$
\hat{z}_{n}(\xi, \xi)=z_{n}(\xi, \xi)=\varphi_{n}(\xi) \text {. }
$$

By $s(n, \zeta)$ we shall denote $\delta\left(\hat{d}_{n, \zeta}\right)$. It is easy to see that for each $\zeta \leqslant \xi$ we have

In particular

$$
s(n, \zeta) \leqslant s(n, \xi) .
$$

Moreover

$$
\mathrm{V}_{\xi \epsilon\langle a, b\rangle}[s(n, \xi) \leqslant s(n, b)] .
$$

Hence

$$
\mathrm{V}_{\eta} \mathrm{H}_{N}[n \geqslant N \Rightarrow s(n, b) \leqslant \eta] \text {. }
$$

$$
\mathrm{V}_{\eta} \mathbb{H}_{N} \mathrm{~V}_{\xi \in\langle a, b\rangle}[n \geqslant N \Rightarrow s(n, \xi) \leqslant \eta] .
$$

From (1.7) and (1.10) it follows that

$$
\mathrm{V}_{e}\left\langle\mathcal{H}_{N} \mathrm{~V}_{\xi \epsilon\langle a, b\rangle} \boldsymbol{V}_{x \epsilon\langle a, b\rangle}\left[n \geqslant N \Rightarrow\left|\hat{z}_{n}(x, \xi)-y(x)\right| \leqslant \varepsilon\right]\right.
$$


and from (1.9) we have

$$
\mathbf{V}_{\varepsilon} \mathbb{H}_{N} \mathbf{V}_{\xi \epsilon\langle a, b\rangle}\left[n \geqslant N \Rightarrow\left|\varphi_{n}(\xi)-y(\xi)\right| \leqslant \varepsilon\right],
$$

which completes the proof of Theorem 1 .

\$ 2. Remark 2. If we assume that $f(x, y)$ has both partial first derivatives, then each $\varphi_{n}(x)$ has a first derivative. We prove this easily by induction with respect to $n$ (making use of (1.4)).

Now the following problem appears: Is the sequence of derivatives $\left\{\varphi_{n}^{\prime}(x)\right\}$ uniformly convergent to the derivative $y^{\prime}(x)$ of the solution of the problem (1.1), (1.2)?

To answer this problem we shall prove the following

THEOREM 2. Let us assume that

1. $f(x, y)$ is defined and continuous in $T$,

2. $f(x, y)$ has both first derivatives, fulfilling the Lipschitz condition with respect to both variables,

3. $|f(x, y)| \leqslant M,\left|\frac{\partial f}{\partial x}\right| \leqslant M_{1},\left|\frac{\partial f}{\partial y}\right| \leqslant M_{2}$,

4. $(b-a) M_{2}<1, b-a<1$.

Then the sequence od derivatives $\left\{\varphi_{n}^{\prime}(x)\right\}$ is uniformly convergent in $\langle a, b\rangle$ to the derivative of the solution of the problem (1.1), (1.2).

Remark 3. From the assumptions of Theorem 2 it follows that in the interval $\langle a, b\rangle$ there exists exactly one solution $y(x)$ of the problem (1.1), (1.2).

Proof. $\mathrm{I}_{2}$. At first we shall show that there exists a number $Q_{1}$ such that

$$
\left|\varphi_{n}^{\prime}(x)\right| \leqslant Q_{1} \quad \text { for each } \quad x \epsilon\langle a, b\rangle, n=0,1, \ldots
$$

We put

$$
\lambda_{n}(x)=a+\frac{n-1}{n}(x-a), \quad \mu_{n}(x)=\varphi_{n-1}\left(\lambda_{n}(x)\right)
$$

Hence

$$
\lambda_{n}^{\prime}(x)=\frac{n-1}{n}, \quad \mu_{n}^{\prime}(x)=\varphi_{n-1}^{\prime}\left(\lambda_{n}(x)\right) \cdot \frac{n-1}{n} .
$$

From (1.4) we have

$$
\begin{aligned}
\varphi_{n}^{\prime}(x)=\mu_{n}^{\prime}(x) & +\frac{1}{n} f\left[\lambda_{n}(x), \mu_{n}(x)\right]+ \\
+ & \frac{x-a}{n}\left\{f_{x}\left[\lambda_{n}(x), \mu_{n}(x)\right] \lambda_{n}^{\prime}(x)+f_{y}\left[\lambda_{n}(x), \mu_{n}(x)\right] \mu_{n}^{\prime}(x)\right\}
\end{aligned}
$$


In view of (2.3) and the assumptions of the theorem, we have

$$
\left|\varphi_{n}^{\prime}(x)\right| \leqslant\left|\varphi_{n-1}^{\prime}\left(\lambda_{n}(x)\right)\right|\left(\frac{n-1}{n}+\frac{b-a}{n} \cdot \frac{n-1}{n} M_{2}\right)+\frac{1}{n} M+\frac{b-a}{n} \cdot \frac{n-1}{n} M_{1} .
$$

Because $(n-1) / n<1$, we have

$$
\left|\varphi_{n}^{\prime}(x)\right| \leqslant\left|\varphi_{n-1}^{\prime}\left(\lambda_{n}(x)\right)\right|\left(1-\frac{1}{n}+\frac{b-a}{n} M_{2}\right)+\frac{1}{n} M+\frac{b-a}{n} M_{1} .
$$

Now we want to find a constant $Q_{1}$ such that

$$
\left\{\left|\varphi_{n-1}^{\prime}(x)\right| \leqslant Q_{1} \text { in }\langle a, b\rangle\right\} \Rightarrow\left\{\left|\varphi_{n}^{\prime}(x)\right| \leqslant Q_{1} \text { in }\langle a, b\rangle\right\} \text {. }
$$

It is easy to see that this condition is fulfilled by each positive solution $Q$ of the following inequality:

$$
Q\left(1-\frac{1}{n}+\frac{b-a}{n}-M_{2}\right)+\frac{1}{n} M+\frac{b-a}{n} M_{1} \leqslant Q
$$

Hence if we put in particular

$$
Q_{1}=\frac{M+(b-a) M_{1}}{1-(b-a) M_{2}}
$$

then $\left|\varphi_{0}^{\prime}(x)\right|=0 \leqslant Q_{1}$ and (2.5) holds, and in consequence (2.1) holds for each $n$.

$\mathrm{II}_{2}$. We shall prove that there exists a constant $R_{1}$ such that

$$
\left|\varphi_{n}^{\prime}(x)-\varphi_{n}^{\prime}(y)\right| \leqslant R_{1}|x-y| \quad \text { for } \quad x, y \in\langle a, b\rangle, n=0,1, \ldots
$$

Let $L_{11}, L_{12}, L_{21}, L_{22}$ be the Lipschitz constants for the partial derivatives of the function $f(x, y)$ (see assumption 2 ), i.e. we have

$$
\begin{aligned}
& \left|f_{x}(x, y)-f_{x}(\bar{x}, \bar{y})\right| \leqslant L_{12}|x-\bar{x}|+L_{12}|y-\bar{y}| \\
& \left|f_{y}(x, y)-f_{y}(\bar{x}, \bar{y})\right| \leqslant L_{21}|x-\bar{x}|+L_{22}|y-\bar{y}|
\end{aligned}
$$

From (2.2) and (2.3) we have

$$
\begin{aligned}
& \left|\lambda_{n}(x)-\lambda_{n}(y)\right| \leqslant \frac{n-1}{n}|x-y| \\
& \left|\mu_{n}(x)-\mu_{n}(y)\right| \leqslant Q_{1} \frac{n-1}{n}|x-y|
\end{aligned}
$$

where $Q_{1}$ is the constant (2.7). From (2.4), (2.9) and (2.10) we infer

$$
\begin{aligned}
& \left|\varphi_{n}^{\prime}(x)-\varphi_{n}^{\prime}(y)\right| \\
& \quad \leqslant \frac{n-1}{n} A_{n}\left|\varphi_{n-1}^{\prime}\left(\lambda_{n}(x)\right)-\varphi_{n-1}^{\prime}\left(\lambda_{n}(y)\right)\right|+\frac{1}{n} \cdot \frac{n-1}{n} B_{n}|x-y|,
\end{aligned}
$$


where

$$
\begin{aligned}
A_{n} & =1+M_{2} \frac{b-a}{n} \\
B_{n} & =2\left(M_{1}+Q_{1} M_{2}\right)+\frac{n-1}{n} C \\
C & =(b-a)\left(L_{11}+L_{12} Q_{1}+L_{21} Q_{1}+L_{22} Q_{1}^{2}\right) .
\end{aligned}
$$

Now we want to find a number $R_{1}$ such that

$$
\left\{\left|\varphi_{n-1}^{\prime}(x)-\varphi_{n-1}^{\prime}(y)\right| \leqslant R_{1}|x-y|\right\} \Rightarrow\left\{\left|\varphi_{n}^{\prime}(x)-\varphi_{n}^{\prime}(y)\right| \leqslant R_{1}|x-y|\right\} .
$$

This property characterizes each positive solution $R$ of the following inequality:

$$
R-R\left(\frac{1}{n}-M_{2} \frac{b-a}{n}\right)+\frac{1}{n}\left[2\left(M_{1}+M_{2} Q_{1}\right)+C\right] \leqslant R .
$$

Hence, if we put in particular

$$
R_{1}=\frac{2\left(M_{1}+M_{2} Q_{1}\right)+C}{1-M_{2}(b-a)}
$$

then $\left|\varphi_{0}^{\prime}(x)-\varphi_{0}^{\prime}(y)\right|=0 \leqslant R_{1}|x-y|$ and (2.15) holds. Hence (2.8) holds for each $n$.

$\mathrm{III}_{2}$. Let $\left\{\varphi_{a_{n}}^{\prime}\right\}$ be an arbitrary subsequence of the sequence $\left\{\varphi_{n}^{\prime}\right\}$. From Arzelo's theorem, the assumptions of which are satisfied in view of parts $\mathrm{I}_{2}$ and $\mathrm{II}_{2}$, it follows that there exists a subsequence $\left\{\varphi_{\beta_{n}}^{\prime}\right\}$ of the sequence $\left\{\varphi_{a_{a}}^{\prime}\right\}$ uniformly convergent. From Theorem 1 it follows that the sequence $\left\{\varphi_{\beta_{n}}\right\}$ is uniformly convergent to $y(x)$. Hence $\left\{\varphi_{\beta_{n}}^{\prime}\right\}$ is uniformly convergent to $y^{\prime}(x)$. But the limit is independent of the choice of the sequence $\left\{\varphi_{a_{n}}^{\prime}\right\}$. Hence $\left\{\varphi_{n}^{\prime}\right\}$ is uniformly convergent in the interval $\langle a, b\rangle$ to the derivative $y^{\prime}(x)$.

\$ 3. Remark 4. It is easy to prove by induction with respect to $n$ that if $f(x, y)$ has all derivatives $\frac{\partial^{p} f}{\partial x^{q} \partial y^{r}}(p=1, \ldots, k, q+r=p$, $q=0,1, \ldots, k, r=0,1, \ldots, k)$, then each $\varphi_{n}(x)$ has all derivatives $\varphi_{n}^{(p)}(x)$ $(p=1, \ldots, k)$.

\section{THEOREM 3. Let us assume that}

1. $f(x, y)$ is defined and continuous in $T$,

2. $|f(x, y)| \leqslant M,\left|\frac{\partial f}{\partial y}\right| \leqslant M_{2}$,

3. $(b-a) \cdot M_{2}<1, b-a<1$,

4. $f(x, y)$ has all bounded partial derivatives $\frac{\partial^{p} f}{\partial x^{q} \partial y^{r}}(p=1, \ldots, k$, $q+r=p, q=0, \ldots, k, r=0, \ldots, k)$, 
5. all derivatives $\frac{\partial^{k} f}{\partial x^{q} \partial y^{r}}(q+r=k, q=0, \ldots, k, r=0, \ldots, k)$ fulfil the Lipschitz condition with respect to both variables.

Then the sequence $\left\{\frac{d^{k}}{d x^{k}} p_{n}(x)\right\}$ is uniformly convergent in $\langle a, b\rangle$ to $\frac{d^{k}}{d x^{k}} y(x)$, where $y(x)$ is the solution of the problem (1.1), (1.2).

Proof. $I_{3}$. From (1.5) it easily follows that for $m \geqslant 2$

where

$$
\varphi_{n}^{(m)}(x)=\frac{m}{n} \sum_{i=0}^{n-1} U_{i}+\frac{x-a}{n} \sum_{i=0}^{n-1} V_{i}
$$

Moreover, it is possible to write

$$
\begin{aligned}
& U_{i}=U_{i}(x)=\frac{d^{m-1}}{d x^{m-1}} f\left[a+\frac{i}{n}(x-a), \varphi_{i}\left(a+\frac{i}{n}(x-a)\right)\right], \\
& V_{i}=V_{i}(x)=\frac{d^{m}}{d x^{m}} f\left[a+\frac{i}{n}(x-a), \varphi_{i}\left(a+\frac{i}{n}(x-a)\right)\right] .
\end{aligned}
$$

where $W_{i}$ is independent of $\varphi_{i}^{(m)}$. Of course $U_{i}$ is also independent of $\varphi_{i}^{(m)}$.

$\mathrm{II}_{3}$. It is possible to prove that for each $m$ there exists a constant $Q_{m}$ such that

$$
\left|\varphi_{n}^{(m)}(x)\right| \leqslant Q_{m} \quad \text { for each } \quad x \epsilon\langle a, b\rangle, n=0,1, \ldots
$$

In order to prove this, we apply the induction procedure with respect to $m$. For $m=0(3.5)$ holds evidently $\left(Q_{0}=|c|+M\right)$. In view of the inequality (2.1) (see part $I_{2}$ of the proof of Theorem 2) it holds also for $m=1$. Now we assume that there exist such constants $Q_{p}(p \leqslant s-1)$ that (3.5) holds for all $m=0,1, \ldots, s-1$. Now, the induction procedure with respect to $n$ proceeds in the same manner as in part $\mathbf{I}_{2}$ of the proof of Theorem 2. In consequence there exists a constant $Q_{s}$, such that (3.5) holds for $m=s$, which finishes the induction proof of (3.5) for all $m$.

$\mathrm{III}_{3}$. Remark 5. If we make the first and the second assumptions of Theorem 1 and, moreover, assume that $f(x, y)$ fulfils the Lipschitz condition with respect to $x$ and $y$, with the constants $K$ and $L$ respectively, then

$$
\left|\varphi_{n}(x)-\varphi_{n}(y)\right| \leqslant \boldsymbol{R}_{0}|x-y|
$$

for each $x \epsilon\langle a, b\rangle, n=0,1, \ldots$, where

$$
R_{0}=\frac{M+(b-a) K}{1-(b-a) L} .
$$


The proof of this follows the same method as the proof of (2.1), Of course, if we suppose that $f(x, y)$ has both bounded partial derivatives, then $f(x, y)$ fulfils the condition of Lipschitz and (3.6) holds.

It is possible to prove that for each $m$ there exists a constant $R_{m}$ such that

$$
\left|\varphi_{n}^{(m)}(x)-\varphi_{n}^{(m)}(y)\right| \leqslant R_{m}|x-y| \quad \text { for } \quad x, y \in\langle a, b\rangle, n=0,1, \ldots
$$

In order to prove the existence of that $R_{m}$ we apply the induction procedure with respect to $m$. In view of Remark 5 and $(2.8),(3.7)$ holds for $m=0,1$.

Now we assume that there exist such constants $R_{p}(p \leqslant s-1)$ that (3.7) holds for $m=0,1, \ldots, s-1$. Then $U_{i}$ and $V_{i}$ are polynomials of the Lipschitz functions and in consequence they are also Lipschitz functions.

Now the induction procedure with respect to $n$ proceeds in the same manner as in part $\mathrm{II}_{2}$ of the proof of Theorem 2 . Hence, we infer the existence of such a constant $R_{s}$ that (3.7) holds for $m=s$, which completes the proof of (3.7) for all $m$.

$\mathrm{IV}_{3}$. In order to finish the proof of the theorem, we apply a similar reasoning to that followed in part $\mathrm{III}_{2}$ of the proof of Theorem 2.

\section{Relerence}

[1] E. Kamke, Differentialgleichungen I, Leipzig 1962.

Reçu par la Rédaction le 1. 8. 1962 